
\title{
PReserch Square \\ Hydroxyapatite Adsorbed at the Surface of the Byssus Fibers of Pinna Nobilis
}

\section{Lorena C. Giannossa}

University of Bari Aldo Moro

Annarosa Mangone

University of Bari Aldo Moro

\section{Giovanni Lagioia}

University of Bari Aldo Moro

Gerardo Palazzo

University of Bari Aldo Moro

Luigi Gentile ( $\sim$ luigi.gentile@uniba.it )

University of Bari Aldo Moro

\section{Research Article}

Keywords:

Posted Date: January 27th, 2022

DOI: https://doi.org/10.21203/rs.3.rs-1280567/v1

License: (c) (1) This work is licensed under a Creative Commons Attribution 4.0 International License. Read Full License 


\section{Abstract}

Mussel-forming fibers manifest fascinating properties due to the numerous reactive functional groups present in their primary structure besides their secondary structures. Humans adopted these proteinbased fibers to produce an extremely fine and valuable fabric, named byssus. Byssus is the bundle of filaments secreted by Pinna nobilis species employed to colonize the marine substrates. The byssus fibers coming from other species, such as Mytilus edulis, have been widely investigated in particular for their self-healing properties attributed to the collagen presence and their core-shell structure. Surprisingly, the Pinna nobilis byssus is non-collagenous based and the fibers nanostructure is still poorly understood. Here, we are reporting an investigation on the Pinna nobilis byssus samples retrieved intact in each stage of treatment. The scanning electron microscope (SEM) provides a peculiar feature of Pinna nobilis byssus, i.e. the elliptical cross-section of the fibers. Wide-angle X-ray scattering (WAXS), as well as infrared spectroscopy (IR), reveal the presence of hydroxyapatite adsorbed at the surface of the initial fibers and the presence of crystalline beta-sheet domains within the fibers. Finally, a WAXS-based method reveals a decrease in the crystalline/amorphous ratio during byssus treatment.

\section{Introduction}

The byssus fibers are protein-based extracellular threads employed by mussels to colonize marine substrates and resist predation. The word "byssus" is the zoological term used to call the fine but strong filaments of fibre beard produced by the sedentary mollusk Pinna nobilis with the aim of fastening itself to the seagrass and withstand the marine currents flow. Pinna nobilis, endemic in the Mediterranean, was the raw material used to make the very fine and valuable sea-silk weaving, but it has been classified as a species of Community Interest in need of strict protection by the European Habitats Directive $(92 / 43 / E E C),{ }_{1}^{1}$ consequently, a renewed production of the beautiful iridescent amber-golden textile is practically impossible. Only a few specific authorizations have been granted over the years to study the bivalve. $^{2}$

The byssus fibers (from mussels) are nowadays known models for biomimetic materials along with silk and other organisms-fabricated protein-based fiber materials. ${ }^{3-5}$ The remarkable mechanical performance and self-healing capacity of byssus fibers produced by other mussel species, in particularly from the genus Mytilus, such as Mytilus edulis, M. californianus, M. trossulus and M. galloprovincialis has attracted a considerable interest in the attempt to understand the relationship between molecular composition and fiber properties. ${ }^{6-8}$ Byssal threads from the Mytilus genus show a similar composition, even though the final threads are not equivalent in their mechanical properties and are characterized by a core-shell structure. ${ }^{9-11}$ In particular, Mytilus byssal threads have a core made of anisotropic bundles of collagen fibers, whereas the shell, known as the cuticle, is a composite of granules dispersed in a continuous, the granules are mainly made of His/3,4-di-hydroxyphenylalanine (DOPA) protein, which, at $\mathrm{pH} 5-8$, readily binds iron to form cross-linking bis- and tris-catecholate-iron complexes. ${ }^{10,12}$ Furthermore, 
a third interlayer region intercalated between the cuticle and core rich in cysteine has been individuated. ${ }^{11}$

In general, for the Mytilus genus, every single fiber consists of an elastic proximal section and a stiff distal section besides the adhesive plaque at their distal end. The proximal and distal sections are both made of pepsin-resistant protein complexes consisting of a collagen core domain, variable flanking regions, and DOPA-rich regions. ${ }^{13,14}$ The flanking regions and DOPA regions are involved in metal ion binding promoting adhesion potential. ${ }^{15,16}$ Notably, histidine's are suspected to play an essential role as sacrificial bonds in yield and self-healing in the distal region of byssal threads. ${ }^{17}$ The distal stiff section is characterized by the presence of mostly $\beta$-structured $(70 \%)$, well-ordered proteins oriented along the fiber axis. ${ }^{18-20}$ Hagenau et al. ${ }^{20}$ demonstrated the structural differences between the proximal and the distal portion by using FTIR and wide-angle X-ray scattering (WAXS).

The byssal threads from other genus species such as Perna perna, Isognomon bicolor, Xenostrobus secures and Brachidontes solisianus are also mainly made of collagen protein ${ }^{6,21}$ although their collagen sequence is species specific. ${ }^{16}$

The Pinna nobilis byssus threads, in contrast to those of the other species, show a paucity of collagen components as demonstrated by several studies. ${ }^{2,6,22}$ In particular, Pinna nobilis'threads, show the lowest Gly content of $14 \%$ of the total amino acid composition with respect to all other species in which ranges between 26 and $41 \%$ and they are also poor in Ala and DOPA, while are particularly rich in Asx, Pro, Tyr, Val, Met, Leu, and Lys. ${ }^{6}$ Bouhlel et al. concluded that the low proportion of DOPA and histidine, and the elevated proportion of Asx, Glx and Lys suggest that the byssus protein assembly could be principally driven by electrostatic interactions, while all the other species rely on the use of collagen as well as amino acid cross-linking with metals, but to different extents. This would explain the lowest mechanical performances of Pinna nobilis'threads with respect to the other species. Moreover, the Pinna nobilis fibers are also characterized by a low diameter of $50 \mu \mathrm{m}$ on average and they are typically a dense bundle of long threads.

Pioneer works on Pinna nobilis'byssus fibers by small and wide-angle synchrotron x-ray scattering (SAXS and WAXS) revealed the less crystalline character with respect to other byssus fibers, ${ }^{23}$ highlighting, in particular, the differences in the recorded diffraction patterns of the threads distal region respect to the Mytilus ones. ${ }^{24}$ Moreover, the presence of Debye-Scherrer diffraction ring was reported for Pinna nobilis'byssus fibers without precise attribution. ${ }^{25,26}$ Pasche et al. ${ }^{2}$ investigated fresh extracted Pinna nobilis'byssus threads by analysing the metal presence and amino acid composition confirming that suchfibers are non-collagenous protein-based proposing that nanofibrils are made of assembled globular protein subunits organized into a helical structure. Pasche et al. ${ }^{2}$ have also investigated the structure of the byssus fibers, by SAXS and WAXS measurements, confirming the absence of the typical collagen peak at $d=0.287 \mathrm{~nm}$ or of any other sharp peaks, while two diffuse haloes were detected from $q=10 \mathrm{~nm}^{-}$ ${ }^{1}$ to $q=25 \mathrm{~nm}^{-1}$. In general, all of the types of collagen contain at least one triple helix ${ }^{27}$, for type I and II 
a radial projection of a single polypeptide chain which has 10 protein motifs in three turns has a translation of $\sim 0.286 \mathrm{~nm}$ which leads to the corresponding WAXS peak based on the description provided by Rich and Crick. ${ }^{28}$ Interestingly, Pasche et al. ${ }^{2}$ did not report any Debye-Scherrer diffraction rings in the WAXS measurements.

However, currently, there is no systematic scientific work regarding the chemical and physical process behind the treatment adopted to produce the byssus fibers for textile applications. Here the scanning electron microscope (SEM) micrographs demonstrated that the byssus stored at the Commodity Science Museum of the University of Bari "Aldo Moro" is coming from Pinna nobilis. Besides, a systematic investigation was conducted by using wide-angle X-ray scattering (WAXS) and infrared spectroscopy $(\mathrm{IR})$ on the fibers collected at each stage of treatment, starting from the raw byssal threads up to the woven fabric. Here taking advantage of the extremely rare documented Pinna nobilis samples, we investigated structural and chemical changes as a function of the treatment process. The treatment process is mainly made of pre-acid wash and acidic washing. We documented the presence of hydroxyapatite adsorbed at the surface of the raw byssus fiber at the initial stage of treatment as well as a decrease in the crystallinity index with the treatment process.

\section{Methods}

Materials

A unique Pinna nobilis sampling is stored at the Commodity Science Museum of the University of Bari Aldo Moro, where all the different phases of treatment of Pinna nobilis fibers are stored. The analyses focused on sea-silk samples starting from the raw byssal threads up to the woven fabric, samples are listed in Table 1.

Table 1. Byssus samples stored at the Commodity Science Museum of the University of Bari Aldo Moro for each stage of the acid-wash treatment. 


\section{Sample $\mathrm{N}^{\circ} \quad$ Stages of treatment}

\begin{tabular}{ll}
\hline $\mathbf{1}$ & Raw Byssus \\
\hline $\mathbf{2}$ & Byssus after first whashings \\
\hline $\mathbf{3}$ & Purged byssus (first phase) \\
\hline $\mathbf{4}$ & Purged byssus (second phase) \\
\hline $\mathbf{5}$ & Dried byssus \\
\hline $\mathbf{6}$ & Byssus ready for carding \\
\hline $\mathbf{7}$ & Carded byssus \\
\hline $\mathbf{8}$ & Yarn byssus \\
\hline $\mathbf{9}$ & Byssus treated after a lemon bath \\
\hline
\end{tabular}

First, the raw byssus collected was subjected to various washes to desalinate and purge it. Second, the fibers were left to nature air-drying. Later, selected byssus was carded and yarned. Lastly, the yarn was immersed in lemon juice for 36 hours.

Wide-angle X-ray scattering (WAXS)

A bundle of the byssus fibers for each sample from sample 1 to sample 11 were located vertically on the sample holder, so-called sandwich cell holder (Hilgenberg $\mathrm{GmbH}$, Malsfeld, Germany) with the fibre direction perpendicular to the X-ray beam. Acquisition times for a WAXS experiment were $2400 \mathrm{~s}$, for the high- $q$ (from $0.13 \mathrm{~nm}^{-1}$ to $3.4 \mathrm{~nm}^{-1}$ ). The bundle were made of several fibers of each sample, i.e. sample amount and thickness were not constant for all samples consequently data are presented in arbitrary units. WAXS measurements were performed using a SAXSLab Ganesha 300XL instrument (SAXSLAB ApS, Skovlunde, Denmark) and a pinhole collimated system equipped with a Genix 3D X-ray source (Xenocs SA, Sassenage, France). The scattering intensity $I(q)$ was recorded with the detector placed at a sample-to-detector distance of $91.742 \mathrm{~mm}$, yielding scattering vectors $(q)$ from $3.5 \mathrm{~nm}^{-1}$ to $27 \mathrm{~nm}^{-1}$. In all cases, the temperature was controlled by an external recirculating water bath fixed to $22^{\circ} \mathrm{C}$, with an accuracy of ca. $0.2^{\circ} \mathrm{C}$. The two-dimensional (2D) scattering pattern was recorded using a $2 \mathrm{D} 300 \mathrm{k}$ Pilatus detector (Dectris Ltd., Baden, Switzerland) and radially averaged using SAXSGui software to obtain $I(q)$. All the WAXS data were normalized on the intensity of the transmitted beam through the sample.

Scanning electron microscope (SEM)

The topography of the same thread was observed using a scanning electron microscope (SEM) LEO EVO50XVP (Zeiss, Cambridge, Cambridgeshire, UK) coupled with an X-max (80 mm2) Silicon drift Oxford detector (Oxford Instruments, High Wycombe, Buckinghamshire, UK) equipped with a Super Atmosphere Thin Window $\odot$. Some fibers of different specimens were fixed on SEM aluminium stubs with carbon 
based double sided adhesive discs and covered with a thin layer of gold using a turbo-pumped sputter coater.

\section{FTIR-ATR.}

The byssus fibers were analysed by FTIR spectroscopy using a Perkin-Elmer UATR Two spectrophotometer equipped with a single reflection diamond ATR crystal (refractive index of 2.4). The spectra were recorded in the range between 750 and $4000 \mathrm{~cm}^{-1}$ with a resolution of $2 \mathrm{~cm}^{-1}$. All the spectra were acquired in 10 scans applying the baseline correction and the ATR correction.

\section{Results And Discussion}

The byssus fibers of Pinna nobilis are characterized by the elliptical shape of the fiber cross-sections, with a greater axis of 30-50 $\mu \mathrm{m}$ and a minor axis of $20 \div 25 \mu \mathrm{m} .{ }^{29}$ The SEM micrographs confirm the external morphology of the raw byssus, sample 1, (Figure 1A) as well as the elliptical cross-section (Figure 1B) of a single fiber, a distinguishing feature of the Pinna nobilis source. The nanostructure changes of those fibers along treatment were investigated by WAXS covering almost three orders of magnitude in the scattering vector, $q$. The treatment stages of byssus fibers examined in this study are mentioned in material methods (Table 1). Briefly, the byssus collected was subjected to washing, drying, carding, yarning and acid washing.

The scattering profile of the investigated samples exhibits a diffuse halo at $\mathrm{q} \sim 14 \mathrm{~nm}^{-1}$ attributed to random coil proteins or also from proteins with a mixture of many secondary structures with various orientations by Pasche et al. ${ }^{2}$ Moreover, on the contrary respect to Pasche et al. ${ }^{2}$ the samples from the initial fibers, raw byssus (sample 1 ) to the purged byssus (sample 4 ) exhibit sharp peaks at $d \approx 0.30$ and $\approx 0.33 \mathrm{~nm}$.

The anisotropic 2D WAXS pattern recorder for the raw material extracted from the mussels, sample 1, contains two Debye-Scherrer diffraction rings (sharp peaks in the 1D profiles) along with arc signals. The crystallites in solid-state materials of macromolecules, such as polymers, are distributed either randomly, in a uniaxial, or partly biaxial distribution in disordered crystalline domains. The uniaxial distribution of crystalline domains in these materials occurs mostly in fibers and the resulting diffraction pattern is the "fiber pattern" in contrast to the random or isotropic distribution of crystalline domains, which resembles a crystalline powder characterized by isotropic diffraction rings resulting in the so-called powder pattern or Debye-Scherrer pattern. ${ }^{30}$ The fiber axis in the fiber pattern is the so-called meridian, while the perpendicular direction is the equator. The partial alignment of the fibers leads to anisotropic arcs in the diffraction pattern. The sharp peak detected for sample 1 at $d \approx 0.30 \mathrm{~nm}$ is not coming from a meridional arc signal i.e. from distance between adjacent amino acid residues in a collagen fiber, while it originated by one of the Debye-Scherrer diffraction rings (Figure 2 ), consequently $d \approx 0.30 \mathrm{~nm}$ could not be assigned to a stretched collagen peak ${ }^{28}$. Moreover, a second sharp peak coming from the second 
Debye-Scherrer diffraction ring is located at $d \approx 0.33 \mathrm{~nm}$. Those reflections are striking similar to the tabulated hydroxyapatite crystal reflections $\mathrm{d}_{002}=0.330-0.348 \mathrm{~nm}$ and $\mathrm{d}_{210}=0.309-0.299 \mathrm{~nm}^{31}$.

In Figure 2, the 0 -to- $360^{\circ}$ azimuthal average profile (azimuthal average), the - 10 -to- $10^{\circ}$ (i.e. along the equatorial direction) and 80 -to- $100^{\circ}$ azimuthal average profiles (i.e. along the meridional direction) are almost identical, considering that, the peak at $d=0.285 \mathrm{~nm}(\mathrm{X}$ in Figure 2$)$ is coming from a single spot and cannot be considered as a structural feature. The meridional signal at $0.38 \mathrm{~nm}$ has been associated with the repeat distance of a $\beta$-pleated sheet. ${ }^{20}$ The halo at $q \sim 14 \mathrm{~nm}^{-1}(d=0.44 \mathrm{~nm})$, is a result of the superposition of amorphous and crystalline components in the byssus fibers, in fact, superposed sharp peaks can be seen in the 1D profiles of Figure 2.

The amorphous peak indicates the presence of disordered structures attributed to random coil proteins or also from proteins with a mixture of many secondary structures by Pasche et al. ${ }^{2}$ It is worth highlighting the amorphous collagen peak was located at $0.46 \mathrm{~nm}$ along with a strong discrete equatorial signal at $1.2 \mathrm{~nm}$ in the distal section of Mytilus galloprovincialis fibers. ${ }^{20}$ In contrast, in the present case of Pinna nobilis, the anisotropic diffraction is located in the meridional direction at $d=1.03 \mathrm{~nm}$ which follows another anisotropic diffraction located at $d=1.32 \mathrm{~nm}$. However, we cannot fully rule out the presence of a very small fraction of collagen in the triple-helical form because the distance between two triple helices is estimated between 1.0 and $1.2 \mathrm{~nm} .{ }^{32}$ On the other hand, the 1D scattering profile obtained from the -10to- $10^{\circ}$ region has low-intensity anisotropic diffraction at $d=0.69 \mathrm{~nm}$ and another one at $d=0.97 \mathrm{~nm}$, confirming our hypothesis related to the presence of hydroxyapatite considering that $a=b=9.415 \AA, c=$ $6.879 \AA$ are the cell parameters of the hexagonal form. ${ }^{33}$

3.2 Chemical substances and functional groups. Figure 3 shows the IR spectra of the Pinna nobilis' byssus fibers for all stages of treatment investigated. These byssus fibres exhibit an amide I band at $1636 \mathrm{~cm}^{-1}$. This band originates from $\mathrm{C}=\mathrm{O}$ stretching with minor contributions from in-plane $\mathrm{N}-\mathrm{H}$ bending and $\mathrm{C}-\mathrm{N}$ stretching within the protein backbone, consequently is greatly affected by the strength of hydrogen bonding and as such is an indicator of the protein secondary structure. ${ }^{34-36}$ In particular the band at $1636 \mathrm{~cm}^{-1}$ is indicating intermolecular parallel $\beta$-sheets presence. ${ }^{36-38}$ The band at $1518 \mathrm{~cm}^{-1}$ is due to amide II, while $\mathrm{C}-\mathrm{H}$ deformation vibrations appear at around $1440 \mathrm{~cm}^{-1}$. On the other hand, collagen triple helix is not detected or their content is negligible since the bands at 1659 and $1555 \mathrm{~cm}^{-1}$ are not predominant. ${ }^{39}$ Figure 3 also shows a broad peak at $3274 \mathrm{~cm}^{-1}$ due to $\mathrm{N}-\mathrm{H}$ stretching vibrations that give rise to amide A. ${ }^{40}$ The narrow signals at around $2932 \mathrm{~cm}^{-1}$ correspond to $\mathrm{C}-\mathrm{H}$ stretching vibrations. Notably, samples from 1 to 4 have higher intensity peat at $1031 \mathrm{~cm}^{-1}$ with respect to the amide peaks, while for all the other samples the relative intensity is lower. The peak at $1031 \mathrm{~cm}^{-1}$ can be attributed to the antisymmetric stretching of $\mathrm{PO}_{4}$, typical of hydroxyapatite. ${ }^{41}$

Mussels' adhesive versatility may lie in the amino acid composition of proteins found near the plaquesubstrate interface which are rich in DOPA and lysine amino acids ${ }^{42}$. It has been shown that the odihydroxyphenolic moiety of DOPA is implicated in the strong H-bonding to hydroxyapatite and 
coordination complexes with the oxides of iron, zinc, titanium, and aluminum. ${ }^{43-46}$ The hydroxyapatite can be synthesized from the mussel shells, ${ }^{47,48}$ nevertheless, there was no previous evidence of hydroxyapatite nanocrystals on the byssus fibers. Here our data are converging on the hydroxyapatite presence on the byssus surface at the initial stages of treatment (from 1 to 4 ).

3.3 Fiber structural changes during treatment. For the samples from 1 to 6 , anisotropic diffraction arcs can be observed clearly indicating a partial alignment of the structures along with the fiber, while from samples 7 to 9 , only the peak at $0.93 \mathrm{~nm}$ is anisotropic but much broader with respect to the corresponding one in previous samples. Moreover, 4 spots have been identified at a very low- $q$ of $4.23 \mathrm{~nm}$ (inset of Figure 4) from samples 5 to 6 . Pasche et al. ${ }^{2}$ attribute the diffraction peaks at $d=4.18 \mathrm{~nm}$ in the small-angle X-ray scattering regime of Pinna nobilis' byssus to a helical superstructure with two protein subunits per turn. Those peaks are not detectable anymore in sample 7. The X-ray fiber measurements revealed a difference in protein structure with a smooth transition from highly ordered structures in the raw material to a less ordered one in the treated material. In the further treatment stages, the amorphous component increases may be due to the stretching and washing procedures overcoming all the other signals. Finally, the last stage leads to much more tight microfibrils, since the meridional peaks completely disappear in favor of a broad peak, while a higher lateral packing of molecules inside the fibrillar structure is noticed since the intensity at the equator is higher.

The two isotropic diffraction rings in the 2D WAXS pattern of sample 1 (sharp peaks) can be also detected in the samples from 1 to 4 . However, the diffraction rings position is not constant. This evidence further corroborates our hypothesis of hydroxyapatite presence adsorbed at the fiber surface, since the mineral size and crystallinity are affected by treatment methods and conditions. For instance, in fresh porcine bones the WAXS signal indicates a low crystallinity, but if treated with supercritical fluid $\mathrm{CO}_{2}$ extraction showed a slightly distinguishable pattern. ${ }^{49}$ Interestingly, a Debye-Scherrer diffraction ring at $\mathrm{d} \approx 0.33 \mathrm{~nm}$ was reported in the pioneering work of Trogus and Hess ${ }^{26}$ along with other diffraction peaks, while Centola reported a peak at $\mathrm{d} \approx 0.45 \mathrm{~nm} .^{25}$

Sample 9 shows a different anisotropic pattern with a preferential alignment along the equator that represent a higher lateral packing of molecules inside the fibrillar structure, even though the amorphous ring dominates the pattern. The Debye-Scherrer diffraction rings disappear in the 2D pattern of sample 5 (Figure 3).

3.4 The crystallinity index of the byssus fibers during treatment. The degree of structural order in the fiber determines the physical and mechanical properties, usually addressed as the degree of crystallinity. The degree of crystallinity can be evaluated by several physical methods that might be affected to different extents by imperfections in crystals and interfacial effects providing some disagreement among the results of quantitative measurements of crystallinity. ${ }^{50}$ The degree of crystallinity of a material is often expressed as the crystallinity index, $C_{i}$ which is the mass ratio of the crystalline substance in the total dry sample based on the crystallographic two-phase model. Here WAXS was adopted to calculate the 
crystallinity index. There are several procedures to calculate the crystallinity index by WAXS mainly developed for polysaccharide-based fibers. ${ }^{50,51}$ These procedures can lead to low crystallinity values due to over-fitting of the amorphous component as it might fail to account for all of the diffraction peaks present under the amorphous halo. Moreover, it should be noticed that the background subtraction might affect the result since the background is a sum of the contribution from the amorphous regions, from incoherent scattering, and scattering from disordered crystallites. See supporting information for further details. The crystallinity index, $\mathrm{C}_{\mathrm{i}}$, was calculated as

$$
C_{i}=1-\frac{\int_{2 \theta_{1}}^{2 \theta_{2}} I_{a m} d 2 \theta}{\int_{2 \theta_{1}}^{2 \theta_{2}} I_{T O T} d 2 \theta}=1-\frac{A_{a m}}{A_{T O T}}
$$

where $I_{a m}$ is the intensity of the fitted amorphous halo (between 13.9 and $13.7 \mathrm{~nm}^{-1}$ ) and $I_{\text {tot }}$ is the total scattering intensity of the sample, including both amorphous and crystalline contributions, $2 \theta_{1}=5.6^{\circ}$ and $2 \theta_{2}=30.7^{\circ}$. The crystallinity index of the raw byssus fiber, sample 1 , is approximately $0.2(20 \%)$ in good agreement with dragline silk fiber usually between 0.22 and $0.24 .^{52}$ The crystallinity index decreases during the treatment process (Figure 5) this might due to the fiber stretching (carding stage). The $C_{i}$ decrease upon stretching is reported for silkworms' fibers indicating a decrease in $\beta$-sheet plate content..$^{53}$ A further decrease in $C_{i}$ takes place in the last stage of treatment (lemon bath) leading to the final sea-silk.

In conclusion, the SEM micrographs identified the byssus fibers of the Pinna nobilis stored at the Commodity Science Museum of the University of Bari Aldo Moro by the elliptical shape of the fiber crosssections. The presence of hydroxyapatite was detected due to the Debye-Scherrer diffraction rings located at $d \approx 0.30 \mathrm{~nm}$ and $0.33 \mathrm{~nm}$ in the WAXS pattern and the antisymmetric stretching of PO4 at $1031 \mathrm{~cm}-1$, corroborated its presence in the initial stage of treatment. The hydroxyapatite powder is supposed to be located on the surface of the byssus threads. Simulations on the mechanism of protein adsorption on the surface of hydroxyapatite are highlight changes in protein orientation, while no changes were observed in protein conformation. 54 Hydroxyapatite removal is obtained by consecutive washing procedures of the treatment process this explains why several authors did not report any Debye-Scherrer diffraction rings for treated byssus. Here a hydroxyapatite shell was found adsorbed on the byssus surface of the Pinna nobilis which is in contrast to the DOPA-Fe shell structure observed for the byssus of the Mytilus. The presence of the $\beta$-sheet plate is confirmed by the feature at $0.38 \mathrm{~nm}$ in the WAXS pattern and by the IR peak at $1636 \mathrm{~cm}-1$ of $\mathrm{C}=0$ stretching within the protein backbone. Finally, the crystallinity index was decreasing during the byssus stages of treatment.

\section{Declarations}

Data availability

All data generated or analysed during this study are included in the supplementary information files.

Competing interests (mandatory) 
The authors declare no competing financial interest.

Acknowledgements

This project has received funding from the European Union's Horizon 2020 research and innovation programme under grant agreement No 731019 (EUSMI). EUSMI was granted to L.G.

Author contributions

A.M. and G.L. provided samples; L.C.G. and L.G. designed research and performed research; L.G. analyzed data; G.P. and L.G. and wrote the paper.

\section{References}

1. EU. EUR-Lex - 31992L0043 - EN. Official Journal L 206, 22/07/1992 P. 0007 - 0050; Finnish special edition: Chapter 15 Volume 11 P. 0114; Swedish special edition: Chapter 15 Volume 11 P. 0114; (1992).

2. Pasche, D. et al. A new twist on sea silk: The peculiar protein ultrastructure of fan shell and pearl oyster byssus. Soft Matter14, 5654-5664 (2018).

3. Kivelio, A. et al. Mussel mimetic tissue adhesive for fetal membrane repair: Initial in vivo investigation in rabbits. European Journal of Obstetrics and Gynecology and Reproductive Biology171, 240-245 (2013).

4. Yang, M., Yamauchi, K., Kurokawa, M. \& Asakura, T. Design of silk-like biomaterials inspired by musseladhesive protein. Tissue Engineering13, 2941-2947 (2007).

5. Liu, Y., Ling, S., Wang, S., Chen, X. \& Shao, Z. Thixotropic silk nanofibril-based hydrogel with extracellular matrix-like structure. Biomaterials Science2, 1338-1342 (2014).

6. Bouhlel, Z. et al. Interspecies comparison of the mechanical properties and biochemical composition of byssal threads. Journal of Experimental Biology220, 984-994 (2017).

7. Sun, C., Lucas, J. M. \& Waite, J. H. Collagen-binding matrix proteins from elastomeric extraorganismic byssal fibers. Biomacromolecules3, 1240-1248 (2002).

8. Waite, J. H. Mussel adhesion - Essential footwork. Journal of Experimental Biology220, 517-530 (2017).

9. Brazee, S. L. \& Carrington, E. Interspecific comparison of the mechanical properties of mussel byssus. Biological Bulletin211, 263-274 (2006).

10. Holten-Andersen, N. et al. Metals and the integrity of a biological coating: the cuticle of mussel byssus. Langmuir: the ACS journal of surfaces and colloids25, 3323-3326 (2009). 
11. Valois, E., Hoffman, C., Demartini, D. G. \& Waite, J. H. The Thiol-Rich Interlayer in the Shell/Core Architecture of Mussel Byssal Threads. Langmuir35, 15985-15991 (2019).

12. Harrington, M. J., Masic, A., Holten-Andersen, N., Waite, J. H. \& Fratzl, P. Iron-clad fibers: a metal-based biological strategy for hard flexible coatings. Science (New York, N. Y.)328, 216-220 (2010).

13. Bell, E. C. \& Gosline, J. M. Mechanical design of mussel byssus: Material yield enhances attachment strength. Journal of Experimental Biology199, 1005-1017 (1996).

14. Benedict, C. V. \& Waite, J. H. Location and analysis of byssal structural proteins of Mytilus edulis. Journal of Morphology189, 171-181 (1986).

15. Lin, Q. et al. Adhesion mechanisms of the mussel foot proteins mfp-1 and mfp-3. Proceedings of the National Academy of Sciences of the United States of America104, 3782-3786 (2007).

16. Qin, X. \& Waite, J. H. Exotic collagen gradients in the byssus of the mussel Mytilus edulis. Journal of Experimental Biology198, (1995).

17. Vaccaro, E. \& Waite, J. H. Yield and post-yield behavior of mussel byssal thread: A self-healing biomolecular material. Biomacromolecules2, 906-911 (2001).

18. Qin, X. X., Coyne, K. J. \& Waite, J. H. Tough tendons. Mussel byssus has collagen with silk-like domains. Journal of Biological Chemistry272, 32623-32627 (1997).

19. Hardy, J. G., Römer, L. M. \& Scheibel, T. R. Polymeric materials based on silk proteins. Polymer49, 4309-4327 (2008).

20. Hagenau, A., Scheidt, H. A., Serpell, L., Huster, D. \& Scheibel, T. Structural analysis of proteinaceous components in byssal threads of the mussel Mytilus galloprovincialis. Macromolecular Bioscience 9 , 162-168 (2009).

21. Harrington, M. \& Waite, J. Short-order tendons: liquid crystal mesophases, metal-complexation and protein gradients in the externalized collagens of mussel byssal threads. in Fibrous Proteins (ed. Scheibel, P. D. T.) 30-45 (Landes Bioscience, 2008). doi:10.1201/9781498713689

22. Jackson, S. F. et al. The bysus threads of Mytilus edulis and Pinna nobilis. in Nature and Structure of COllagen (eds. Randall, J. T. \& Jackson, F. R. S.) London, Bu, 106-116 (Butterworths Scientific Publications, 1953).

23. Mercer, E. H. Observations on the molecular structure of byssus fibres. Marine and Freshwater Research3, 199-205 (1951).

24. Rudall, K. The distribution of collagen and chitin. Fibrous proteins and their biological significance. Symphosia of the Society for Experimental Biology 49-71 (1955). 
25. Centola. Struttura e proprietà del 'bisso' o seta di ostriche. Gazzetta chimica italiana66, 71-80 (1936).

26. Trogus, C. \& Hess, K. Zur Kenntnis der natürlichen Seiden und ihres Verhaltens gegen Säuren und Basen. Biochem. Z.260, 376 (1933).

27. Ricard-Blum, S. The Collagen Family. Cold Spring Harbor Perspectives in Biology3, 1-19 (2011).

28. Rich, A. \& Crick, F. H. C. The molecular structure of collagen. Journal of Molecular Biology3, 483-506 (1961).

29. Diana, A. et al. The byssus threads of pinna nobilis: A histochemical and ultrastructural study. European Journal of Histochemistry61, 280-296 (2017).

30. Zugenmaier, P. X-ray analysis of partial crystalline fibre structure. in Handbook of Textile Fibre Structure1, 46-120 (Elsevier Ltd, 2009).

31. Giannini, C. et al. Scanning SAXS-WAXS microscopy on osteoarthritis-affected bone - An age-related study. in Journal of Applied Crystallography47, 110-117 (International Union of Crystallography, 2014).

32. Terzi, A. et al. Effects of processing on structural, mechanical and biological properties of collagenbased substrates for regenerative medicine. Scientific Reports8, (2018).

33. Procopio, A. et al. Chemical Fingerprint of Zn-Hydroxyapatite in the Early Stages of Osteogenic Differentiation. ACS Central Science5, 1449-1460 (2019).

34. Chalmers, J. \& Griffiths, P. Handbook of Vibrational Spectroscopy, 5 volumes set. (2002).

35. Jackson, M. \& Mantsch, H. H. The use and misuse of FTIR spectroscopy in the determination of protein structure. Critical Reviews in Biochemistry and Molecular Biology30, 95-120 (1995).

36. Byette, F., Marcotte, I. \& Pellerin, C. Covalently crosslinked mussel byssus protein-based materials with tunable properties. Peptide Science111, e24053 (2019).

37. Chen, F., Porter, D. \& Vollrath, F. Silk cocoon (Bombyx mori): Multi-layer structure and mechanical properties. Acta Biomaterialia8, 2620-2627 (2012).

38. De Meutter, J. \& Goormaghtigh, E. Evaluation of protein secondary structure from FTIR spectra improved after partial deuteration. European Biophysics Journa/1, 3 (2021).

39. Belbachir, K., Noreen, R., Gouspillou, G. \& Petibois, C. Collagen types analysis and differentiation by FTIR spectroscopy. Analytical and Bioanalytical Chemistry395, 829-837 (2009).

40. Barth, A. \& Zscherp, C. What vibrations tell us about proteins. Quarterly Reviews of Biophysics35, 369-430 (2002). 
41. Eslami, H., Solati-Hashjin, M., Tahriri, M. \& Bakhshi, F. Synthesis and characterization of nanocrystalline hydroxyapatite obtained by the wet chemical technique. Materials Science- Poland28, 5$13(2010)$.

42. Lee, H., Dellatore, S. M., Miller, W. M. \& Messersmith, P. B. Mussel-inspired surface chemistry for multifunctional coatings. Science318, 426-430 (2007).

43. McWhirter, M. J., Bremer, P. J., Lamont, I. L. \& McQuillan, A. J. Siderophore-mediated covalent bonding to metal (oxide) surfaces during biofilm initiation by pseudomonas aeruginosa bacteria. Langmuir19, 3575-3577 (2003).

44. Ramakrishna, G. \& Ghosh, H. N. Effect of particle size on the reactivity of quantum size ZnO nanoparticles and charge-transfer dynamics with adsorbed catechols. Langmuir19, 3006-3012 (2003).

45. Liu, Y., Dadap, J. I., Zimdars, D. \& Eisenthal, K. B. Study of interfacial charge-transfer complex on TiO2 particles in aqueous suspension by second-harmonic generation. Journal of Physical Chemistry B103, 2480-2486 (1999).

46. Simpson, S. L., Powell, K. J. \& Sjöberg, S. Pyrocatechol violet complexation at the boehmite-water interface. Journal of Colloid and Interface Science229, 568-574 (2000).

47. Shavandi, A., Bekhit, A. E. D. A., Ali, A. \& Sun, Z. Synthesis of nano-hydroxyapatite (nHA) from waste mussel shells using a rapid microwave method. Materials Chemistry and Physics149, 607-616 (2015).

48. Edralin, E. J. M., Garcia, J. L., dela Rosa, F. M. \& Punzalan, E. R. Sonochemical synthesis, characterization and photocatalytic properties of hydroxyapatite nano-rods derived from mussel shells. Materials Letters196, 33-36 (2017).

49. Barakat, N. A. M. et al. Physiochemical characterizations of hydroxyapatite extracted from bovine bones by three different methods: Extraction of biologically desirable HAp. Materials Science and Engineering C28, 1381-1387 (2008).

50. Ahvenainen, P., Kontro, I. \& Svedström, K. Comparison of sample crystallinity determination methods by X-ray diffraction for challenging cellulose I materials. Cellulose23, 1073-1086 (2016).

51. Asaadi, S. et al. Structural analysis of Ioncell-F fibres from birch wood. Carbohydrate Polymers181, 893-901 (2018).

52. Yazawa, K., Malay, A. D., Masunaga, H. \& Numata, K. Role of Skin Layers on Mechanical Properties and Supercontraction of Spider Dragline Silk Fiber. Macromolecular Bioscience19, 1800220 (2019).

53. Numata, K. et al. Use of extension-deformation-based crystallisation of silk fibres to differentiate their functions in nature. Soft Matter11, 6335-6342 (2015). 
Figures
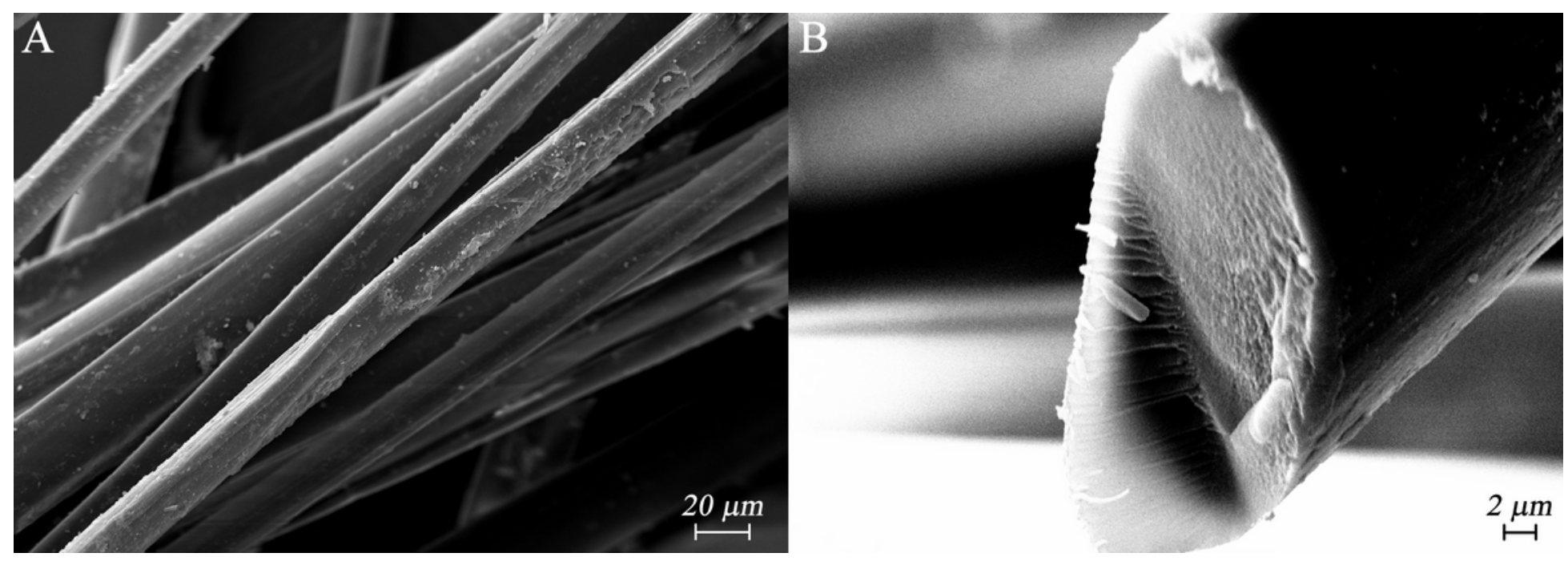

Figure 1

SEM micrograph of the raw byssus (sample 1) fibres bundle (A) and the cross-section of one fiber (B) from the species Pinna nobilis.
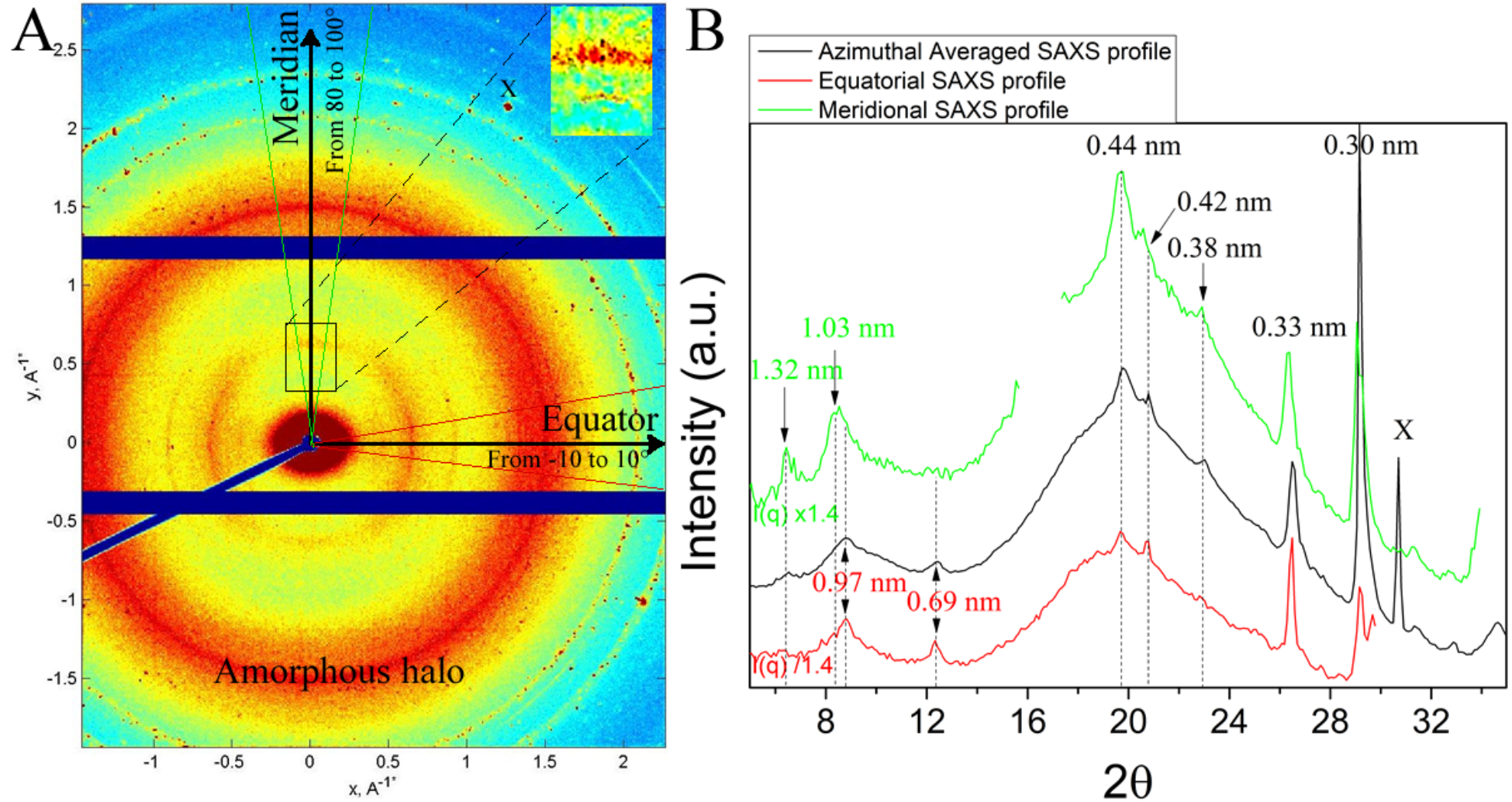

Figure 2

(A) The 2D WAXS pattern for the raw fibers extracted from the mussels (sample 1) in which isotropic diffraction rings, amorphous halo and anisotropic diffraction can be observed. The inset in is highlighting 
the 2D signal in the meridional direction close to the beam stop. (B) The 1D WAXS profiles were obtained upon 0 -to- $360^{\circ}$ (Azimuthal averaged), -10 -to-10 (Equatorial) and 80-to-100 (Meridional) averaging the $2 \mathrm{D}$ patterns. $\mathrm{X}$ is a high-intensity spot not related to structural features.

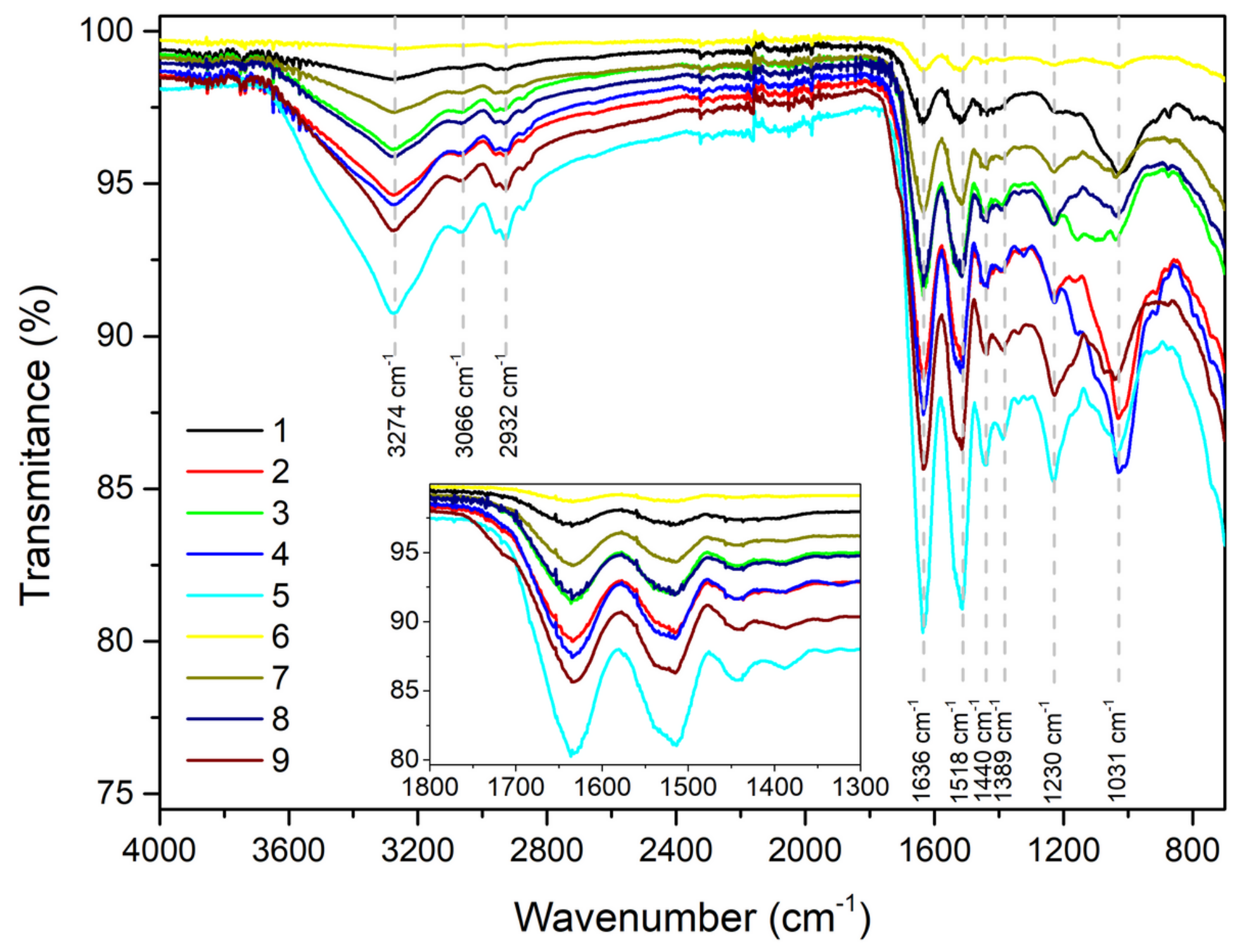

Figure 3

FTIR spectra of the byssus fibers of the Pinna nobilis at all stages of treatment investigated. 

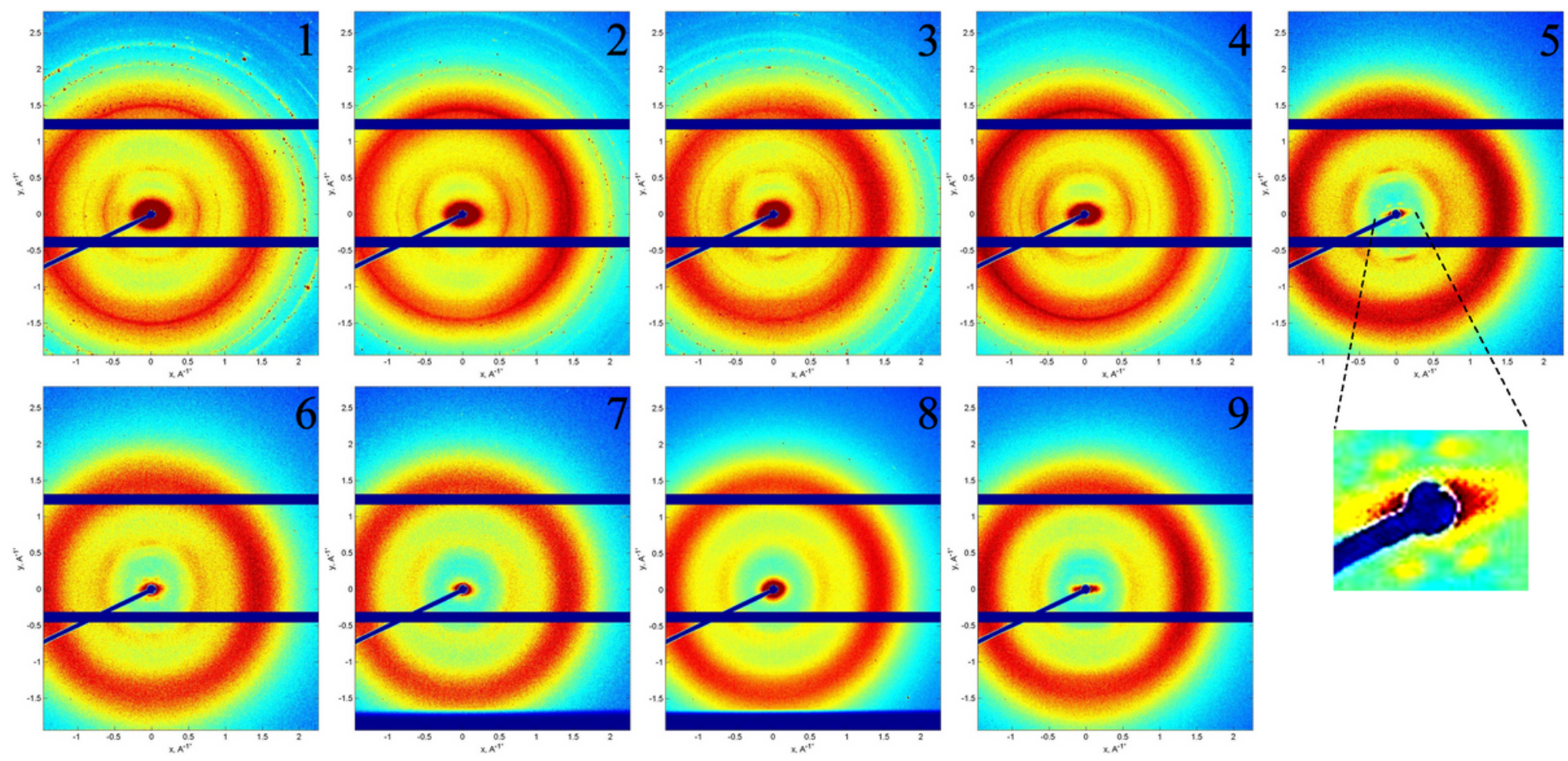

Figure 4

2D WAXS pattern for the byssus fibers of the Pinna nobilis from the first (sample 1) to the last (sample 9) stages of treatment. The inset is a zoom at low-q for sample 5, where (at $4.18 \mathrm{~nm}$ ) 4 spots can be seen. 


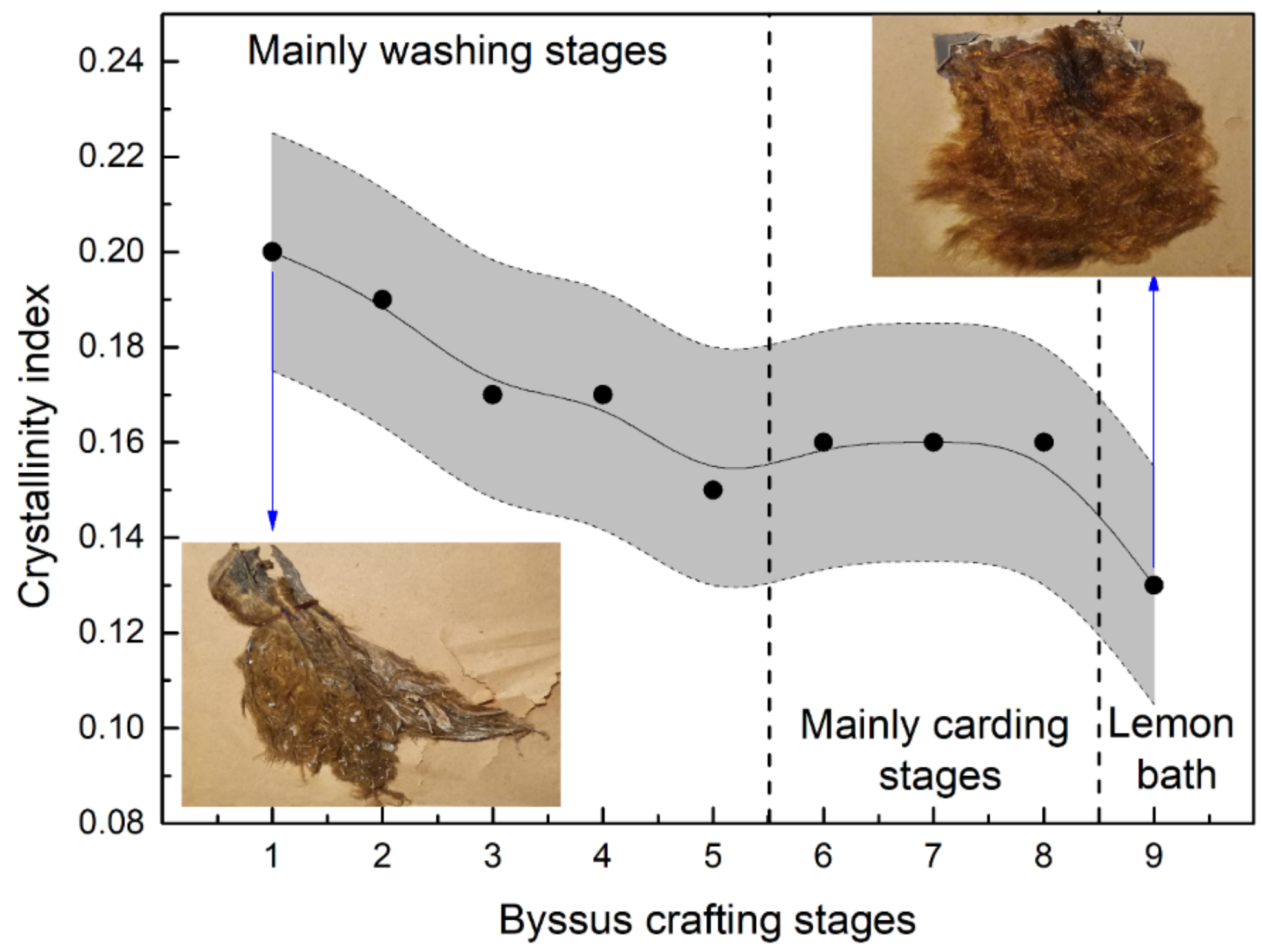

Figure 5

The crystallinity index of byssus during the stages of treatment obtained by WAXS. The grey region represents the error bar. The insets are photographs of the raw byssus (sample 1) and the byssus treated after a lemon bath (sample 9).

\section{Supplementary Files}

This is a list of supplementary files associated with this preprint. Click to download.

- PinnaNobilisGentileScientificReportsSI.docx 